\author{
Monique van Dijk \\ Hennie Knoester \\ Babette S. van Beusekom \\ Erwin Ista
}

\section{Screening pediatric delirium with an adapted version of the Sophia Observation withdrawal Symptoms scale (SOS)}

Accepted: 29 October 2011

Published online: 9 December 2011

(C) The Author(s) 2011. This article is published with open access at

Springerlink.com

\section{Dear Editor}

Pediatric delirium (PD) has an estimated incidence of $4-16.9 \%$. Three motoric subtypes are distinguished: hyperactive, hypoactive, and mixed. Current screening scales used are the Delirium Rating Scale (DRS) [1], the Pediatric Anesthesia Emergence Delirium Scale (PAED) [2], and the pCAM-ICU [3]. These scales are not well suited for the PICU setting, however, either because they have not been validated for this setting or are age inappropriate.

Our group validated the Sophia Observation withdrawal Symptoms scale to assess withdrawal symptoms in critically ill children [4]. As withdrawal syndrome is a possible cause of delirium, we assumed that symptoms of both conditions may overlap.

To confirm this, we retrieved medical and nursing records of 29 patients (12 girls) admitted to two PICUs in The Netherlands from January 2001 to March 2009 with a child and adolescent psychiatrist-confirmed diagnosis of delirium. Table 1 gives the demographic characteristics. Two researchers (MvD, EI) independently documented all symptoms suspected to indicate delirium recorded in the period from $48 \mathrm{~h}$ before to $48 \mathrm{~h}$ after the psychiatrist's consultation. Discrepancies were discussed until consensus was reached. The result was 19 different symptoms, 10 of which are included in the SOS (Table 2). Five of the 19 symptoms correspond with the major delirium symptoms in the DSM-IV [5]. Almost
Table 1 Characteristics of 29 patients with delirium

\begin{tabular}{ll}
\hline Variable & $N(\%)$ \\
\hline Sex (male/female) & $17 / 12$ \\
Age in years & \\
Reason for admission & $12(2-18)$ (IQR 8-16.5) \\
Trauma & $11(37.9 \%)$ \\
Cardiac & $7(24.1 \%)$ \\
Respiratory & $3(10.3 \%)$ \\
Oncology & $2(6.9 \%)$ \\
Infectious & $2(6.9 \%)$ \\
Hemolytic uremic syndrome & $1(3.4 \%)$ \\
Hypertensive crisis & $1(3.4 \%)$ \\
Metabolic disregulation & $1(3.4 \%)$ \\
Mesenteric artery thrombosis & $1(3.4 \%)$ \\
Type of admission medical/surgical & $17 / 12$ \\
Mechanical ventilation & $23(79 \%)$ \\
PIM 2 score & $3.6(0.8-35.2)$ \\
PRIMS II score & $12(0-23)$
\end{tabular}

$I Q R$ Interquartile range

a Median (minimum-maximum)

Table 2 Symptoms of delirium reported by nurses and psychiatrists $(N=29$ patients)

\begin{tabular}{llll}
\hline Symptom & $\begin{array}{l}\text { Occurrence } \\
(\%)\end{array}$ & In & Major delirium \\
& SOS & symptoms DSM-IV
\end{tabular}

Agitation

$25(86.2) \times$

Irritable

Pulls out lines, tubes, or catheters

Fidgety, restless, or jittery

Hallucinations (visual or auditory)

Anxiety

Motor disturbance

$22(75.8)$

Increased muscle tension (e.g., muscle cramp) 3 (10.3)

Grimacing

Tremors (e.g., shivery)

Decreased or varying consciousness

$2(6.9)$

Confusion

e.g., (trying to) get out of bed

Disorientation

Time

Place

Person

Sleep disturbance

Impaired or confused speech

Seizures

Pupil dilation

$5(17.2)$

$16(55.2)$

$16(55.2)$

$15(51.7)$

$8(27.6)$

$12(41.4)$

$3(10.3)$

$12(41.3) \times$

$9(31.0) \times \times($ crit. B $)$

Itching

$2(6.9)$

$2(6.9)$

$2(6.9)$

$1(3.4)$

Forgetfulness/impaired memory $1(3.4)$

Hyperacusis

$1(3.4)$

Sweating

$1(3.4)$

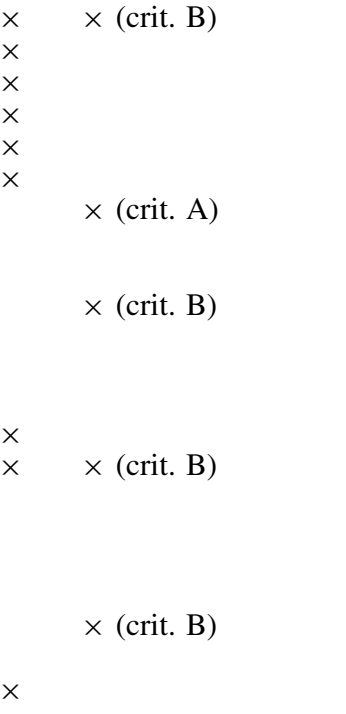

${ }^{a}$ Not documented: acute onset, fluctuations (crit. C). Fluctuations are reflected in varying scores on the extended SOS-Pediatric Delirium scale 
all patients showed agitation, defined as irritability; attempts to pull out lines, tubes, or catheters; restlessness or fidgeting. Twenty-two patients $(76 \%)$ showed hallucinations, mostly visual hallucinations.

Only a few symptoms suggested hypoactive delirium, such as decreased consciousness, impaired memory, and attentional deficits. We propose that adding these symptoms to the SOS, as well as lethargy and decreased motor activity, would make the SOS sensitive for hypoactive delirium as well. However, if hypoactive delirium is suspected from the observed symptoms, a child and adolescent psychiatrist should make a differential diagnosis to exclude a depressive disorder or adjustment disorder with anxiety or depression. Furthermore, hypoactive delirium in children must be differentiated from regressive behavior, stress, or a dissociative/conversion disorder.

We think the way to validate the extended SOS scale is to suspect delirium if at least one of the items from category B (far right column, Table 2) is observed. The acute onset and fluctuations of symptoms (e.g., sleepy during the daytime and delirious during the night) typical for delirium require repeated assessments $24 \mathrm{~h}$ per day.

Limitations of this study are its retrospective nature and the small sample size. Furthermore, preverbal children were underrepresented, perhaps because hallucinations and hypoactive delirium are difficult to diagnose in the very young $[6,7]$. This could mean that the real incidence of delirium in these patients may be much higher than now observed in daily practice. Future studies, therefore, should focus on detecting delirium in preverbal children. One way to go is to ask parents of young children with suspected delirium how the child's behavior compares to the usual behavior. Future studies should also find better ways to identify hypoactive delirium and its risk factors. Next, RCTs could identify the most effective pharmacological treatment for delirium in children.

Open Access This article is distributed under the terms of the Creative Commons Attribution Noncommercial License which permits any noncommercial use, distribution, and reproduction in any medium, provided the original author(s) and source are credited.

\section{References}

1. Turkel SB, Braslow K, Tavare CJ, Trzepacz PT (2003) The delirium rating scale in children and adolescents. Psychosomatics 44:126-129

2. Janssen NJ, Tan EY, Staal M, Janssen EP, Leroy PL, Lousberg R, van Os J, Schieveld JN (2011) On the utility of diagnostic instruments for pediatric delirium in critical illness: an evaluation of the Pediatric Anesthesia Emergence Delirium Scale, the Delirium Rating Scale 88, and the Delirium Rating ScaleRevised R-98. Intensive Care Med 37:1331-1337

3. Smith HA, Boyd J, Fuchs DC, Melvin K, Berry P, Shintani A, Eden SK, Terrell MK, Boswell T, Wolfram K, Sopfe J, Barr FE, Pandharipande PP, Ely EW (2011) Diagnosing delirium in critically ill children: Validity and reliability of the Pediatric Confusion Assessment Method for the Intensive Care Unit. Crit Care Med 39:150-157
4. Ista E, van Dijk M, de Hoog M, Tibboel D, Duivenvoorden HJ (2009) Construction of the Sophia Observation withdrawal Symptoms-scale (SOS) for critically ill children. Intensive Care Med 35:1075-1081

5. American Psychiatric Association (2000) Diagnostic and statistical manual of mental disorders : DSM-IV-TR. American Psychiatric Association, Washington

6. Madden K, Turkel S, Jacobson J, Epstein D, Moromisato DY (2012) Recurrent delirium after surgery for congenital heart disease in an infant. Pediatr Crit Care Med 13

7. Silver GH, Kearney JA, Kutko MC, Bartell AS (2010) Infant delirium in pediatric critical care settings. Am J Psychiatry 167:1172-1177

M. van Dijk · E. Ista (ه)

Intensive Care, Erasmus MC-Sophia Children's Hospital, P.O. Box 2060, 3000 CB Rotterdam, The Netherlands e-mail: w.ista@erasmusmc.nl

Tel.: +31-10-7037028

Fax: +31-10-7036796

\section{H. Knoester}

Pediatric Intensive Care Unit,

Emma Children's Hospital,

Amsterdam Medical Center,

Amsterdam, The Netherlands

B. S. van Beusekom

Department of Child and Adolescent Psychiatry, Erasmus MC-Sophia Children's Hospital, Rotterdam, The Netherlands 